\title{
ASSOCIATION BETWEEN PRE-STROKE PHYSICAL ACTIVITY AND MOBILITY AND WALKING ABILITY IN THE EARLY SUBACUTE PHASE: A REGISTRY-BASED STUDY
}

Malin REINHOLDSSON, MSc ${ }^{1,2}$, Anna GRIMBY-EKMAN, PhD ${ }^{3}$ and Hanna C. PERSSON, PhD ${ }^{1,2}$

From the ${ }^{1}$ Institute of Neuroscience and Physiology, Department of Clinical Neuroscience, Sahlgrenska Academy, University of Gothenburg, Gothenburg, Sweden, '2Department of Occupational Therapy and Physiotherapy, Sahlgrenska University Hospital Gothenburg, Sweden and ${ }^{3}$ Biostatistics, School of Public Health and Community Medicine, Institute of Medicine, Sahlgrenska Academy, University of Gothenburg, Gothenburg, Sweden

Objective: To investigate associations between prestroke physical activity and mobility, walking ability, and self-perceived upper extremity function during stroke unit care.

Design: A longitudinal, registry-based study with a consecutively collected cohort.

Subjects/patients: A total of 1,092 adults with stroke admitted to 3 Swedish stroke units between 2017 and 2018.

Methods: Logistic mixed effects regression models were performed to investigate associations (adjusted for age and sex). Pre-stroke physical activity was assessed with Saltin-Grimby Physical Activity Level Scale on admission. Mobility, walking ability, and self-perceived upper extremity function were assessed at admission and discharge from the stroke units and compared between pre-stroke physically active $(45 \%)$ and inactive $(55 \%)$ groups.

Results: All groups of patients showed improvements in mobility $(p<0.001)$, walking ability $(p<0.001)$, and upper extremity function $(p<0.001)$. The changes over time tended to differ between the physically inactive and active groups for mobility $(p<0.062)$ and walking ability $(p<0.056)$, but the differences were not significant.

Conclusion: Pre-stroke physically active people showed a tendency to be more independent in physical functioning early after stroke. Regardless of prestroke physical activity, all patients showed improvements in mobility, walking ability, and self-perceived upper extremity function during inpatient care.

Key words: prestroke; stroke; exercise; physical activity; mobility; transfers; walking; upper extremity.

Accepted Oct 1; Epub ahead of print Oct 15, 2021

J Rehabil Med 2021; 53: jrm00233

Correspondence address: Malin Reinholdsson, Institute of Neuroscience and Physiology, Department of Clinical Neuroscience, Sahlgrenska Academy, University of Gothenburg, Gothenburg, Sweden. E-mail: malin.reinholdsson@vgregion.se, Malin.reinholdsson@gu.se

$\mathrm{T}$ he number of people living with the consequences of a stroke has increased over time (1), which has substantially increased the global burden of adult disability (2). The overall goal of stroke rehabilitation is to regain independence (2), but it has been shown that

\section{LAY ABSTRACT}

To be physically active prior to a stroke may improve the post-stroke recovery process. Therefore, we hypothesized that pre-stroke physical activity might reduce the consequences of a stroke. This study investigated associations between pre-stroke physical activity and post-stroke recovery of mobility, walking ability, and arm and hand function. A total of 1,092 patients were examined; $44 \%$ were women, $89 \%$ had ischaemic stroke, and $55 \%$ were physically inactive before the stroke. All patients showed improvements during care at the stroke units, regardless of their previous physical activity level. At hospital discharge, $71 \%$ of patients showed independent mobility, $68 \%$ could walk independently, and $55 \%$ reported self-perceived arm and hand impairments. Patients with higher pre-stroke physical activity levels had a tendency of being more independent in mobility and walking compared with inactive patients. However, the frequencies of self-perceived arm and hand impairments were similar between the physically active and physically inactive groups. To be physically active prior to a stroke may improve the post-stroke recovery process.

$43 \%$ have remaining disability one month after stroke, and $39 \%$ after 5 years (3). Recovery can be defined as an improvement over time, evaluated as the return to pre-stroke functions and activities, or alternatively, evaluated in terms of the underlying mechanisms (4). Agreed definitions of phases in stroke recovery are acute (1-7 days), early ( 7 days to 3 months), and late (3-6 months) subacute, and chronic (more than 6 months) (4). Improvements mainly occur in the early subacute phase after a stroke (4), and care at a stroke unit is important for positive outcomes (5). Stroke-unit care and rehabilitation increases the probability that patients will survive, return to their own homes, and regain independence (5) at one year post-stroke (6). In stroke rehabilitation research, stroke-related impairments (sensorimotor deficiencies, quality of life, and global disability) should be evaluated with measures capturing mobility, walking, and motor function (7). According to the International Classification of Functioning, Disability, and Health (ICF) (8), mobility and walking ability are categorized as "activities and participation", and upper extremity (UE) function is included in the category of "body structures". 
Pre-stroke physical activity may promote neuroprotective mechanisms, such as angiogenesis, and neuroplasticity, in both human and animal subjects (9). This could contribute to better motor function, and motor recovery (9). Previous studies have shown that physical activity could reduce the risk of stroke by $25-30 \%$ (10). Physical activity is defined as any bodily movement produced by skeletal muscles that requires energy expenditure (11). Different types of physical activity can be categorized as occupational, sports, conditioning, household, or other activities (11). Exercise, which is a subcategory of physical activity, is planned, structured, and repetitive, and its purpose is to improve or maintain physical fitness (11). Pre-stroke physical activity was previously related to a reduction in the size of a cerebral infarction (12), less severe acute stroke symptoms (12-15), less post-stroke disability $(12,15,16)$, and improved performance in activities of daily living (ADL) $(15,17,18)$. Previous studies on disability after stroke often included a mobility assessment, but, typically, mobility was not measured separately from other abilities. Moreover, few studies have focused on associations between pre-stroke physical activity and post-stroke physical functioning (19) (i.e. mobility, walking ability, and UE function). However, 3 small studies showed that pre-stroke physical activity was positively related to post-stroke improvements in balance, walking speed (17), independent gait (20), and walking frequency (21). No studies have investigated the association between pre-stroke physical activity and UE function. Overall, little evidence is available on associations between pre-stroke physical activity and different post-stroke consequences, and the published results are conflicting $(19,22)$. The current study aimed to investigate associations between pre-stroke physical activity and post-stroke mobility, walking ability, and self-perceived UE function during inpatient stroke unit care.

\section{METHODS}

This registry-based study has a longitudinal design. The cohort comprised all patients admitted to 3 comprehensive stroke units at the Sahlgrenska University Hospital, Gothenburg, Sweden, from 31 August 2017 to 31 August 2018. The university hospital includes 3 hospitals and provides emergency and basic care for 700,000 inhabitants in Gothenburg, as well as specialized care for 1.7 million inhabitants in the west of Sweden (Västra Götaland). According to the National strokeregistry, $95 \%$ of the patients received care at the stroke units and 5\% received care elsewhere (23). The inclusion criteria were: data registered in the local quality registry (Väststroke), ischaemic stroke (International Classification of Diseases $10^{\text {th }}$ Revision (ICD-10) code I63) or intracerebral haemorrhage (ICD-10 code I61), and a complete assessment of pre-stroke physical activity. When patients had recurrent strokes within the study period, only the first stroke incident was included. This article was written according to Strengthening the Reporting of Observational Studies in Epidemiology (STROBE) guidelines.

\section{Data acquisition procedure}

Data were collected in a clinical setting by registered nurses, physiotherapists, and occupational therapists. These data were retrieved from medical records and the Väststroke registry. Demographic and medical variables (age, sex, stroke type, stroke severity, need of community care, and death during stroke unit care) were recorded in the registry by nurses at discharge. Stroke severity was assessed with National Institutes of Health Stroke Scales (NIHSS) on admission. Higher NIHSS scores (range 0-42) indicated worse stroke severity $(24,25)$. The scores were categorized as: mild stroke $(0-5)$, moderate stroke (6-14), severe stroke (15-24), and very severe stroke $(\geq 25)(25)$.

Pre-stroke physical activity levels were assessed by the physiotherapist on admission (within $48 \mathrm{~h}$ ) with the question: "How physically active were you before your stroke? Try to estimate an average over the year." This information was self-reported by the patient, and when necessary, confirmed by relatives. The 4-level Saltin-Grimby Physical Activity Level Scale (SGPALS) (26) categorizes leisure-time physical activity, as follows (27):

Physically inactive: nearly completely inactive, occupied with reading, watching television, watching movies, using computers, or performing other sedentary activities.

Some physical activity (light physical activity): physically active for at least $4 \mathrm{~h} /$ week with activities like riding a bicycle or walking to work, walking with the family, gardening, fishing, table tennis, bowling etc.

Regular physical activity and training (moderate physical activity): performing activities like heavy gardening, running, swimming, playing tennis, badminton, calisthenics, and similar activities, for at least $2-3 \mathrm{~h} /$ week.

Regular strenuous physical training for competition sports (vigorous physical activity): performing activities like running, orienteering, skiing, swimming, soccer, European handball etc., several times per week.

\section{Physical functioning outcomes}

The 3 outcome variables in the present study were mobility, walking ability, and stroke-related UE function. Assessments were performed and recorded at 3 time-points: prior to the stroke (data collected on admission), upon admission (within $48 \mathrm{~h}$ ), and at discharge from the stroke unit. Mobility was defined as all indoor transfers between lying, sitting, and standing positions or to and from a bed, chair, or wheelchair. Independence in mobility was assessed by the physiotherapist (yes/no). Walking ability was defined as the ability to walk independently for $10 \mathrm{~m}$ or more indoors, and walking aids were allowed. Independence in walking ability was assessed by the physiotherapist (yes/no). UE function was defined as self-perceived stroke-related arm and hand impairments, which included assessments of strength, sensation, or oedema. UE function (yes/no) was recorded by an occupational therapist. Thus, all outcome variables were binary.

\section{Usual care at stroke units}

Patients admitted to the stroke units received early goaloriented rehabilitation and task-specific training (28) according to the Swedish National Guidelines (29). Assessments were performed routinely (30). Physiotherapists and occupational therapists performed early assessments (within $48 \mathrm{~h}$ ) for $93 \%$ of patients and daily interventions for approximately $84 \%$ of patients (23). The median length of stay at the stroke unit was 8.5 days (23). 


\section{Statistical analysis}

All statistical analyses were performed with IBM SPSS Statistics 25.0 (IBM Corp, Armonk, NY, USA). The level of significance was set at $p<0.05$ and all tests were 2-tailed. Differences between groups were analysed with independent samples $t$-tests (for age), $\chi^{2}$ tests (for sex), and Mann-Whitney $U$ tests (for stroke severity). For descriptive data on pre-stroke physical activity, SGPALS levels 3 and 4 were merged, due to the small number of cases in category $4(n=3)$. Furthermore, for the regression models the scale was dichotomized as physically inactive and physically active (levels $2-4$ were merged) due to lower number of observations at higher SGPALS levels.

Logistic mixed effects regression models, with a random intercept, were performed to investigate the association between prestroke physical activity and each of the binary outcomes (mobility, walking ability, and UE function) over time. This modelling was performed to manage the dependence between sequential observations, due to repeated measures over time (31). We represented time in terms of 3 time-points: pre-stroke, admission, and discharge. In the regression model, pre-stroke physical activity and time were included separately, as main effects, and together, as an interaction effect. The interaction term was retained in the regression model when it achieved $p \leq 0.10$. Estimated model probabilities and confidence intervals $(95 \% \mathrm{CI})$ are reported for each model. Models were analysed both unadjusted and after confounder adjustment. The possible confounders age and sex were included in the model, when they achieved $p \leq 0.20$. We could not adjust the models for stroke severity, due to excessive missing values $(n=229)$. Each model was analysed twice: $(i)$ the total sample including patients who died during the stroke unit care $(n=1,092)$, where their data after death is missing observations, (ii) the sample of stroke survivors $(n=983)$, where the non-survivors were deleted.

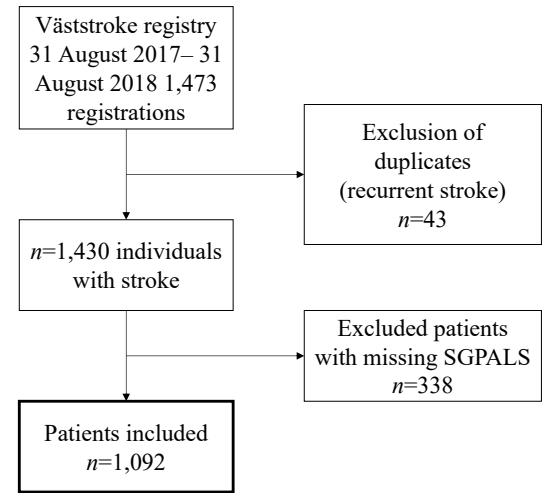

Fig. 1. Flow chart of the patient selection process. SGPALS: SaltinGrimby Physical Activity Level Scale.

\section{RESULTS}

A total of 1,430 patients with stroke were identified in the registry. Of these, 1,092 patients were included in the current study (Fig. 1). The study included only the first stroke for 43 individuals who had experienced recurrent strokes within the study period. A total of 338 patients were excluded due to missing assessments of pre-stroke physical activity. Compared with the included patients, the excluded patients were older ( $p=0.04$, mean age 75.9 years, standard deviation (SD) $13.9)$ and had more severe stroke $(p<0.001$; median NIHSS: 11, interquartile range (IQR): 19-3). The in-

Table I. Descriptive characteristics of patients with different pre-stroke physical activity (PA) levels, according to the Saltin-Grimby Physical Activity Level Scale (SGPALS)

\begin{tabular}{|c|c|c|c|c|}
\hline \multirow{2}{*}{$\begin{array}{l}\text { Characteristics } \\
n(\%)\end{array}$} & \multicolumn{4}{|c|}{ Physically inactive SGPALS 1 Light PA SGPALS 2 Moderate \& Vigorous PA SGPALS $3+4$ Total } \\
\hline & $600(54.9)$ & $427(39.1)$ & $65(6.0)$ & $1,092(100)$ \\
\hline Age, years, mean (SD) & $77.5(12.3)$ & $70.8(13.0)$ & $65.7(14.0)$ & $74.2(13.3)$ \\
\hline Sex, women, $n(\%)$ & $314(52.3)$ & $153(35.8)$ & $18(27.7)$ & $485(44.4)$ \\
\hline At discharge in need of community care, $n(\%)$, missing $=8$ & $240(40.3)$ & $91(21.5)$ & $8(12.9)$ & $339(31.3)$ \\
\hline \multicolumn{5}{|c|}{ Stroke type $n(\%)$, missing $=1$} \\
\hline Cerebral haemorrhage & $67(11.2)$ & $44(10.3)$ & $6(9.2)$ & $117(10.7)$ \\
\hline Ischaemic stroke & $533(88.8)$ & $382(89.5)$ & $59(90.1)$ & $974(89.2)$ \\
\hline \multicolumn{5}{|l|}{ Stroke severity NIHSS $n(\%)$, missing $=229$} \\
\hline Mild stroke & $278(63.8)$ & $267(72.0)$ & $47(83.9)$ & $592(68.6)$ \\
\hline Moderate stroke & $98(22.5)$ & $63(17.0)$ & $7(12.5)$ & $168(19.5)$ \\
\hline Severe stroke & $51(11.7)$ & $39(10.5)$ & $2(3.6)$ & $92(10.7)$ \\
\hline Very severe stroke & $9(2.1)$ & $2(0.5)$ & 0 & $11(1.3)$ \\
\hline \multicolumn{5}{|l|}{ Independent mobility, $n(\%)$} \\
\hline Previously, missing $=55$ & $483(88.3)$ & $423(99.3)$ & $64(100)$ & $970(93.5)$ \\
\hline On admission, missing $=12$ & $220(37.0)$ & $282(66.8)$ & $45(70.3)$ & $547(50.6)$ \\
\hline At discharge, missing $=111$ & $302(57.4)$ & $333(84.5)$ & $56(91.8)$ & $691(70.4)$ \\
\hline \multicolumn{5}{|l|}{ Independent walking, $n(\%)$} \\
\hline On admission, missing $=14$ & $188(31.7)$ & $241(57.2)$ & $45(70.3)$ & $474(44.0)$ \\
\hline At discharge, missing $=106$ & $287(54.5)$ & $326(81.9)$ & $55(90.2)$ & $668(67.7)$ \\
\hline \multicolumn{5}{|l|}{ Self-perceived impaired upper extremity function, $n(\%)$} \\
\hline Previously, missing = 195 & $37(8.0)$ & $26(7.0)$ & $1(1.6)$ & $64(7.1)$ \\
\hline On admission, missing $=152$ & $357(71.3)$ & $257(67.8)$ & $42(70.0)$ & $656(69.8)$ \\
\hline At discharge, missing $=256$ & $262(58.6)$ & $170(50.3)$ & $26(51.0)$ & $458(54.8)$ \\
\hline \multicolumn{5}{|l|}{ Complications during inpatient stroke unit care, $n(\%)$} \\
\hline New stroke, missing $=7$ & $20(3.4)$ & $11(2.6)$ & $1(1.5)$ & $32(2.9)$ \\
\hline Registered falls, missing $=12$ & $61(10.3)$ & $29(6.9)$ & $6(9.4)$ & $96(8.9)$ \\
\hline Deceased during stroke unit care & $93(15.5)$ & $15(3.5)$ & $1(1.5)$ & $109(10.0)$ \\
\hline
\end{tabular}

NIHSS: National Institutes of Health Stroke Scale score (range 0-42); categories: mild: 0-5, moderate: 6-14, severe: 15-24, very severe: 25-42. Valid percentages are presented. 
Table II. Logistic mixed effects regression model results show the effects of 3 variables (pre-stroke physical activity (PA), time, and the interaction between PA and time) on mobility, walking ability, and self-perceived upper extremity (UE) function in patients who experienced a stroke $(n=983)$

\begin{tabular}{llll}
\hline & & $\begin{array}{l}\text { Unadjusted } \\
\text { model }\end{array}$ & $\begin{array}{l}\text { Adjusted } \\
\text { model }\end{array}$ \\
\cline { 3 - 4 } & Variables & $p$-values & $p$-values \\
\hline Mobility & Pre stroke PA & $<0.001$ & $<0.001$ \\
& Time & $<0.001$ & $<0.001$ \\
Walking ability & Pre-stroke PA*Time & 0.062 & 0.074 \\
& Pre stroke PA & $<0.001$ & $<0.001$ \\
UE function & Time & $<0.001$ & $<0.001$ \\
With interaction & Pre-stroke PA*Time & 0.056 & 0.066 \\
UE function & Time & 0.219 & - \\
No interaction & Pre-stroke PA*Time & 0.755 & - \\
\hline
\end{tabular}

Mobility and walking ability were adjusted for age. Sex was not a confounder in the data.

cluded and excluded groups had similar distributions of $\operatorname{sex}(p=0.130)$.

Among the patients included, 109 died during inpatient care, and of these, $85.3 \%$ were physically inactive. In the study population, $44.4 \%$ were women, $89.2 \%$ had ischaemic stroke, and the median NIHSS score was 2 (IQR 8-1). A majority of patients (68.6\%) had experienced a mild stroke (NIHSS 0-5; Table I). Prior to the stroke, $600(54.9 \%)$ patients were physically inactive, $93.5 \%$ reported independent mobility, and $92.8 \%$ were able to walk.

Independent mobility was observed in $50.6 \%$ of patients on admission and $70.4 \%$ at discharge; an
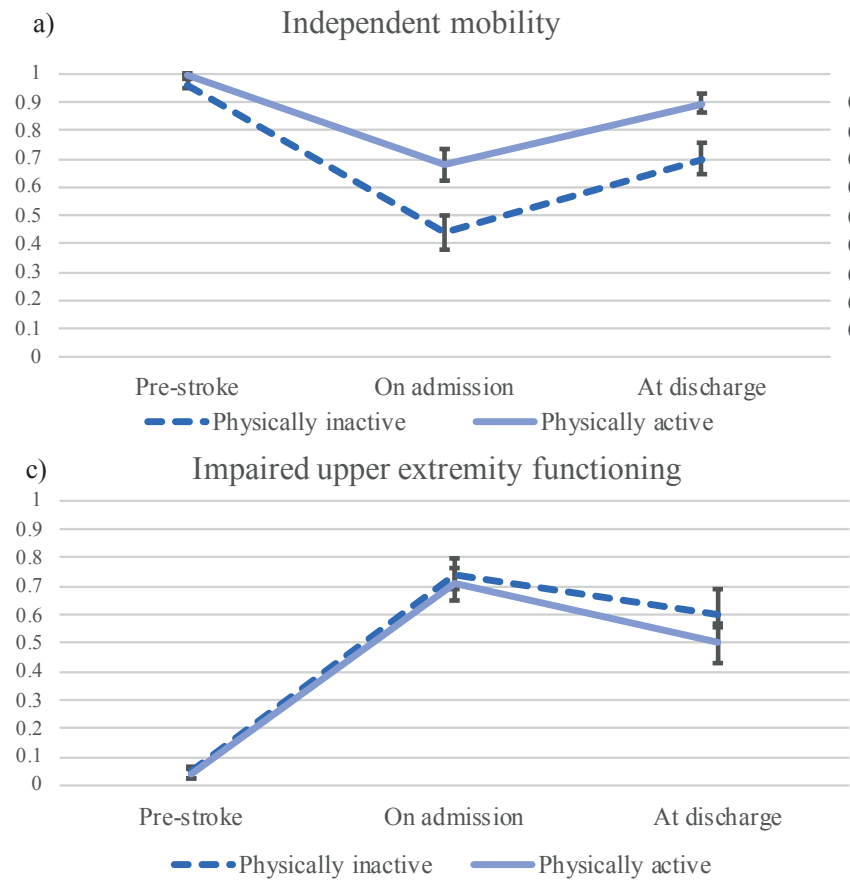

b) Independent walking ability

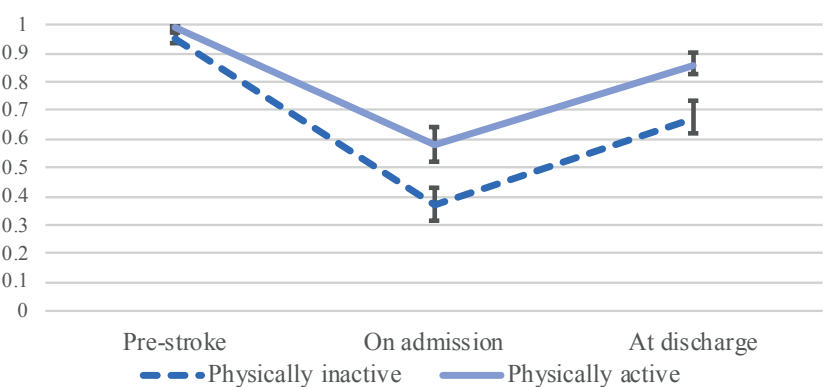

Fig. 2. Changes over time in the probabilities of successful recovery for patients who were physically inactive or physically active before a stroke (adjusted for age). Recovery was measured in terms of: (a) independent mobility, (b) independent walking ability, and (c) self-perceived impaired upper extremity function. Y-axis shows the estimated probabilities of success for each of the outcomes, including $95 \%$ confidence intervals (95\% CI). Values are shown in Appendix I. improvement of $19.8 \%$. Independent walking ability was observed in $44.0 \%$ of patients on admission and $67.7 \%$ at discharge; an improvement of $23.7 \%$ (Table I). In addition, self-perceived impaired UE function was reported by $7.1 \%$ of patients before the stroke, by $69.8 \%$ of patients on admission, and by $54.8 \%$ of patients at discharge; an improvement of $15 \%$.

The interaction terms analysed with logistic mixed effects regression showed that, during inpatient care, mobility, walking ability, and UE function followed similar patterns of improvement over time, regardless e and active groups for mobility and walking ability, the differences were not significant. The interaction for both mobility and walking ability, but $p>0.1$ for UE function. In a model without the interaction term, only time $(p<0.001)$ had a significant effect on UE function.

Between the pre-stroke time and admission, the physically active group did not decline as much as the inactive group (adjusted values for age, as sex was not a confounder, Fig. 2a and b). Although both groups showed improvements at discharge, the active group showed better mobility and walking ability than the inactive group. However, the differences observed between the inactive and active groups were not significant. Furthermore, UE function showed similar patterns of decline

An additional logistic mixed effect model was performed, which included individuals who had died of pre-stroke physical activity (Table II). The changes over time tended to differ between the physically inac- 
during the inpatient stroke unit care. The (unadjusted) results for the logistic mixed effect models that included individuals who had died during the inpatient stroke unit care showed significant differences in mobility $(p=0.022)$ and walking ability $(p=0.014)$ between the physically active and inactive groups for the interaction effect of physical activity and time. However, self-perceived UE function was not significantly different between groups $(p=0.623)$. The estimates for all 3 outcomes were similar between physically active and inactive groups in both analyses: stroke survivors $(n=983)$, and in the total study sample $(n=1,092)$.

\section{DISCUSSION}

This study showed that, during inpatient care at the stroke unit, in the early subacute phase after a stroke, all groups of patients improved in mobility, walking ability, and self-perceived UE function, regardless of the level of pre-stroke physical activity. There was a tendency for patients in the physically active group to show greater improvement in mobility and walking ability, compared with inactive patients. However, improvements in UE function were similar between the physically active and inactive groups. Moreover, patients in the pre-stroke physically active group showed independence in mobility and walking ability, at both admission and at discharge more often compared with patients in the inactive group.

The finding that pre-stroke physically active patients tended to show independence in walking ability was consistent with results from 3 smaller studies $(17,20$, $21)$. One study showed that pre-stroke walk routines with durations that exceed $30 \mathrm{~min} /$ walk were associated with a faster walking speed and better balance in the acute phase (2-6 days) and at one year post-stroke (17). Furthermore, mild or intense pre-stroke physical activity was associated with an independent gait poststroke, although age and a previous stroke showed more important associations (20). Nevertheless, prestroke physical activity was associated with better walking outcomes at 3 and 6 months post-stroke (21). The current study described physical functioning in a sample of patients during the early subacute phase after a stroke. The patients received care at comprehensive stroke units with access to reperfusion therapies. The current study cohort showed less pre-stroke dependence in mobility and walking ability (16-17\%), compared with previous studies that included more patients with premorbid disabilities $(21-33 \%)(3,32)$. At discharge, approximately two-thirds of the current study cohort showed independence in mobility and walking, and approximately half of the cohort reported self-perceived impaired UE function. In comparison, more patients were independent when walking compared with an international study in which $41 \%$ of the patients showed independent walking ability at 7 days after stroke (5), while independent mobility had similar results as other studies in which $40 \%$ of the patients were disabled 1 month to 5 years after a stroke (28) and $70 \%$ with regained motor function in 3-6 months post-stroke (2). Of note, comparison with the latter studies was limited, because those patients were evaluated in the late sub-acute and chronic phases after a stroke (4). Furthermore, in the present study, $69 \%$ of patients had a mild stroke (NIHSS 0-5), which was consistent with National Swedish data $(63 \%$ with a mild stroke) (23), but somewhat inconsistent with the large AVERT study, which included 53\% of patients with a mild stroke (NIHSS 1-7) (5). In the stroke unit, early, frequent rehabilitation interventions that target the patients' needs and goals are essential, and they should be performed during the critical time period to optimize improvement (33). It is likely that patients from the present study will experience improvements after the stroke unit stay and for many patients, rehabilitation will continue.

Physical activity may contribute to neuroprotective effects with increased cerebral blood flow, collateral circulation, and increased growth factor production after cerebrovascular diseases (34). It remains unknown whether neuroprotective mechanisms are associated with better stroke outcomes (35). Based on the current results, pre-stroke physical activity tended to be associated with independent mobility and walking ability, but not self-perceived UE function, both on admission and at discharge. In addition, the current study analysed the interaction between time and the level of physical activity. Although some differences between the pre-stroke physically active and inactive groups were found, the differences were not significant. The lack of association between pre-stroke physical activity and UE function might be explained by the prioritization of mobility and walking activities during acute rehabilitation, by both patients and rehabilitation professionals. Furthermore, the SGPALS assessment of pre-stroke physical activity is more closely related to mobility and walking ability than to UE function. Therefore, the scores might not have reflected prestroke activities that depended on UE function. An instrument that measured pre-stroke arm and hand activity might have been more appropriate.

A major strength of this registry-based study was the large consecutive sample from a taxfunded healthcare database. Thus, the results of the current study are generalizable to similar stroke unit care contexts. A further strength of this study was the early, sub-acute setting after stroke. It has been noted that more research 
should be conducted during the early period after a stroke, because most previous studies were initiated at least 6 months post-stroke (33). This study had some limitations. First, the self-reported, retrospectively collected physical activity assessments were at risk of recall bias. Furthermore, although the SGPALS included several aspects of physical activity, such as duration, intensity, and frequency, it did not reflect work activities, and was dichotomized in the analyses. A more reliable method for assessing physical activity would be to equip patients with accelerometers; however, this method might not be feasible in a large study sample. Another limitation was the mixed outcomes with both objective assessments (mobility, and walking ability) and self-perceived symptoms (UE function). In addition, information may be lost when using dichotomized variables; e.g. there is a large variation between insufficient dexterity and total UE paresis. Although we strived to capture a stroke-unit cohort, the representativeness of the study sample was limited, because a high proportion of patients with severe stroke had not been routinely assessed for pre-stroke physical activity, which could induce selection bias. Therefore, those patients could not be included in the present study. This lack might be explained by a low prioritization of the pre-stroke physical activity level in patients who present with severe stroke or patients in palliative care. Moreover, in the present study, the patients who died during inpatient care were often older, female, inactive, more dependent prior to the stroke, and presented with severe stroke. To control for selection bias, we performed an additional regression analysis that included patients who had died during the study period. That analysis showed similar results. In addition, the study design was inherently limited, due to the pre-set variables in registry-based studies. It would have been interesting to analyse risk factors, such as hypertension, smoking, alcohol use, and diabetes; however, risk factors were not available in the Väststroke registry. Lastly, data available in registries are collected from clinical settings, which can result in missing values. For example, NIHSS scores for stroke severity were missing in $21 \%$ of the patients in the current study.

Future studies on pre-stroke physical activity could be improved by using a prospective study design and objective measurements for physical activity that would be feasible for large populations. To prioritize promotion of increased physical activity can reduce diseases and thereby the global economic burden (36). The current study contributed to knowledge about associations between pre-stroke physical activity and physical functioning in the field of stroke rehabilitation. In addition, this study provided a description of physical functioning, in terms of mobility, walking ability, and self-perceived UE function, in the early subacute period after a stroke.

In conclusion, early after a stroke, all patients in this study who received inpatient care in stroke units showed improvements in mobility, walking ability, and self-perceived UE function. Moreover, the results showed that pre-stroke physically active people had a tendency to be more independent in mobility and walking ability, but not in self-perceived UE function, compared with pre-stroke physically inactive people.

\section{ACKNOWLEDGEIMENTS}

The authors acknowledge Elina Larsson and Caroline Risp from the Physiotherapy program at the University of Gothenburg for writing the first version of the study. We would also like to acknowledge the Väststroke Collaboration for contributing data to the study from the Väststroke Registry, the included patients for participating, and the assisting staff for help with data acquisition.

The study was approved by the Regional Ethical Review Board in Gothenburg, 4 May 2016 (346-16). An additional application was approved on 19 September 2018 (T807-18). This study was conducted in accordance with the World Medical Association Declaration of Helsinki.

Funding. This study was funded by the Local Research and Development Board for Gothenburg and Södra Bohuslän, the Foundation for Neurological research (Insamlingsstiftelsen), and the trust funds from: Ahl \& Wennerström, Greta and Einar Askers, Hjalmar Svensson, and Renée Eander.

The authors have no conflicts of interest to declare.

\section{REFERENCES}

1. Feigin VL, Norrving B, Mensah GA. Global burden of stroke. Circ Res 2017; 120: 439-448.

2. Stinear CM. Prediction of motor recovery after stroke: advances in biomarkers. Lancet Neurol 2017; 16: 826-836.

3. Luengo-Fernandez R, Paul NL, Gray AM, Pendlebury ST, Bull LM, Welch SJ, et al. Population-based study of disability and institutionalization after transient ischemic attack and stroke: 10-year results of the Oxford Vascular Study. Stroke 2013; 44: 2854-2861.

4. Bernhardt J, Hayward KS, Kwakkel G, Ward NS, Wolf SL, Borschmann K, et al. Agreed definitions and a shared vision for new standards in stroke recovery research: the stroke recovery and rehabilitation roundtable taskforce. Int J Stroke 2017; 12: 444-450.

5. Langhorne $\mathrm{P}$, Wu O, Rodgers $\mathrm{H}$, Ashburn A, Bernhardt J. A Very Early Rehabilitation Trial after stroke (AVERT): a phase III, multicentre, randomised controlled trial. Health Technology Assessment (Winchester, England) 2017; 21: 1-120.

6. Stroke Unit Trialists C. Organised inpatient (stroke unit) care for stroke. The Cochrane database of systematic reviews 2013; 2013: CD000197.

7. Kwakkel G, Lannin NA, Borschmann K, English C, Ali M, Churilov $L$, et al. Standardized measurement of sensorimotor recovery in stroke trials: Consensus-based core recommendations from the Stroke Recovery and Rehabilitation Roundtable. Int J Stroke 2017; 12: 451-461.

8. World Health Organization. ICF : International Classification of Functioning, Disability and Health. Geneva: World Health 
Organization; 2001.

9. Pin-Barre C, Laurin J. Physical exercise as a diagnostic, rehabilitation, and preventive tool: influence on neuroplasticity and motor recovery after stroke. Neural Plast 2015; 2015: 608581.

10. Gallanagh S, Quinn TJ, Alexander J, Walters MR. Physical activity in the prevention and treatment of stroke. ISRN Neurol 2011; 2011: 953818.

11. Dasso NA. How is exercise different from physical activity? A concept analysis. Nurs Forum 2019; 54: 45-52.

12. Ricciardi AC, Lopez-Cancio E, Perez de la Ossa N, Sobrino T, Hernandez-Perez M, Gomis M, et al. Prestroke physical activity is associated with good functional outcome and arterial recanalization after stroke due to a large vessel occlusion. Cerebrovasc Dis 2014; 37: 304-311.

13. Reinholdsson M, Palstam A, Sunnerhagen KS. Prestroke physical activity could influence acute stroke severity (part of PAPSIGOT). Neurology 2018; 91: e1461-e1467.

14. Krarup LH, Gluud C, Truelsen T, Pedersen A, Lindahl M, Hansen $L$, et al. The ExStroke pilot trial: rationale, design, and baseline data of a randomized multicenter trial comparing physical training versus usual care after an ischemic stroke. Contemp Clin Trials 2008; 29: 410-417.

15. Deplanque D, Masse I, Lefebvre C, Libersa C, Leys D, Bordet R. Prior TIA, lipid-lowering drug use, and physical activity decrease ischemic stroke severity. Neurology 2006; 67: 1403-1410.

16. Krarup LH, Truelsen T, Gluud C, Andersen G, Zeng X, Korv J, et al. Prestroke physical activity is associated with severity and long-term outcome from first-ever stroke. Neurology 2008; 71: 1313-1318.

17. Ursin $\mathrm{MH}$, Ihle-Hansen $\mathrm{H}$, Fure $B$, Tveit A, Bergland A. Effects of premorbid physical activity on stroke severity and post-stroke functioning. J Rehabil Med 2015; 47: 612-617.

18. Stroud N, Mazwi TM, Case LD, Brown RD, Jr., Brott TG, Worrall $\mathrm{BB}$, et al. Prestroke physical activity and early functional status after stroke. J Neurol Neurosurg Psychiatry 2009; 80: 1019-1022.

19. Tumasz MT, Trocoli T, de Oliveira MF, Campos RR, Botelho RV. do physically active patients have better functional outcome after stroke? A systematic review. J Stroke Cerebrovasc Dis 2016; 25: 527-532.

20. Yamaguchi T, Yamamura O, Hamano T, Murakita $K$, Nakamoto Y. Premorbid physical activity is modestly associated with gait independence after a stroke: an exploratory study. Eur Rev Aging Phys Act 2018; 15: 18.

21. Mahendran N, Kuys SS, Brauer SG. Which impairments, activity limitations and personal factors at hospital discharge predict walking activity across the first 6 months poststroke?
Disabil Rehabil 2020; 42: 763-469.

22. Kramer SF, Hung SH, Brodtmann A. The impact of physical activity before and after stroke on stroke risk and recovery: a narrative review. Curr Neurol Neurosci Rep 2019; 19: $28-28$.

23. The Swedish stroke register (Riksstroke). Annual report for the full year 2018. 2019 [cited 2020 May 15]. Available from: www.riksstroke.se. (In Swedish).

24. Kasner SE. Clinical interpretation and use of stroke scales. Lancet Neurol 2006; 5: 603-612.

25. Lindley RI, Wardlaw JM, Whiteley WN, Cohen G, Blackwell L, Murray GD, et al. Alteplase for acute ischemic stroke: outcomes by clinically important subgroups in the Third International Stroke Trial. Stroke 2015; 46: 746-756.

26. Saltin B, Grimby G. Physiological analysis of middle-aged and old former athletes. Comparison with still active athletes of the same ages. Circulation 1968; 38: 1104-1115.

27. Rodjer L, Jonsdottir IH, Rosengren A, Bjorck L, Grimby G, Thelle DS, et al. Self-reported leisure time physical activity: a useful assessment tool in everyday health care. BMC Public Health 2012; 12: 693.

28. Hankey GJ. Stroke. Lancet 2017; 389: 641-654.

29. National Board of Health and Welfare in Sweden. National guidelines for stroke care. Support for governance and management. 2020. [cited 2020 May 15]. Available from: https:// www.socialstyrelsen.se/regler-och-riktlinjer/nationellariktlinjer/riktlinjer-och-utvarderingar/stroke/. (in Swedish).

30. Alt Murphy M, Björkdahl A, Forsberg-Wärleby G, Persson CU. Implementation of evidence-based assessment of upper extremity in stroke rehabilitation: from evidence to clinical practice. J Rehabil Med 2021; 53: jrm00148.

31. Agresti A. Categorical data analysis. New York: Wiley, 1990.

32. Ganesh A, Luengo-Fernandez R, Pendlebury ST, Rothwell PM. Long-term consequences of worsened poststroke status in patients with premorbid disability. Stroke 2018; 49: 2430-2436.

33. Stinear C, Ackerley S, Byblow W. Rehabilitation is initiated early after stroke, but most motor rehabilitation trials are not: a systematic review. Stroke 2013; 44: 2039-2045.

34. Wang Y, Li M, Dong F, Zhang J, Zhang F. Physical exerciseinduced protection on ischemic cardiovascular and cerebrovascular diseases. Int J Clin Exp Med 2015; 8: 19859.

35. Hatakeyama M, Ninomiya I, Kanazawa M. Angiogenesis and neuronal remodeling after ischemic stroke. Neural regeneration research 2020; 15: 16-19.

36. Ding $D$, Lawson KD, Kolbe-Alexander TL, Finkelstein EA, Katzmarzyk PT, Van Mechelen W, et al. The economic burden of physical inactivity: a global analysis of major noncommunicable diseases. The Lancet 2016; 388: 1311-1324.

Appendix I. Change over time for people with stroke with pre-stroke physical inactivity vs physical activity analysed with logistic mixed effects regression models $(n=983)$.

\begin{tabular}{|c|c|c|c|c|}
\hline \multirow{2}{*}{$\begin{array}{l}\text { Functioning } \\
\text { Time of assessment }\end{array}$} & \multicolumn{2}{|c|}{ Physically inactive estimated probabilities ( $95 \% \mathrm{CI}$ ) } & \multicolumn{2}{|c|}{ Physically active estimated probabilities ( $95 \% \mathrm{CI})$} \\
\hline & Unadjusted & Adjusted & Unadjusted & Adjusted \\
\hline \multicolumn{5}{|l|}{ Mobility } \\
\hline Pre-stroke & $0.95(0.923-0.963)$ & $0.96(0.937-0.970)$ & $0.996(0.988-0.999)$ & $0.996(0.988-0.999)$ \\
\hline On admission & $0.41(0.350-0.466)$ & $0.44(0.380-0.501)$ & $0.71(0.650-0.757)$ & $0.68(0.625-0.737)$ \\
\hline At discharge & $0.66(0.604-0.718)$ & $0.70(0.643-0.754)$ & $0.90(0.859-0.922)$ & $0.89(0.849-0.916)$ \\
\hline \multicolumn{5}{|l|}{ Walking ability } \\
\hline Pre-stroke & $0.95(0.933-0.963)$ & $0.95(0.928-0.965)$ & $0.996(0.988-0.997)$ & $0.99(0.983-0.997)$ \\
\hline On admission & $0.34(0.285-0.393)$ & $0.37(0.310-0.425)$ & $0.61(0.548-0.665)$ & $0.58(0.518-0.639)$ \\
\hline At discharg & $0.63(0.548-0.665)$ & $0.67(0.606-0.720)$ & $0.87(0.830-0.901)$ & $0.86(0.818-0.893)$ \\
\hline \multicolumn{5}{|c|}{ Self-perceived upper extremity functioning } \\
\hline Pre-stroke & $0.05(0.033-0.073)$ & $0.05(0.034-0.075)$ & $0.04(0.025-0.060)$ & $0.04(0.025-0.059)$ \\
\hline On admission & $0.735(0.678-0.785)$ & $0.74(0.682-0.790)$ & $0.72(0.656-0.767)$ & $0.71(0.650-0.764)$ \\
\hline At discharge & $0.57(0.505-0.638)$ & $0.60(0.510-0.644)$ & $0.51(0.436-0.573)$ & $0.50(0.430-0.569)$ \\
\hline
\end{tabular}

95\% CI: 95\% confidence interval. Physical inactivity (SGPALS 1), physically active (SGPALS 2-4), SGPALS: Saltin Grimby Physical Activity Level Scale. Adjusted for age (sex was not a confounder in the data). 